Original Article

\title{
HAART THERAPY IN GHANA: COMPARATIVE ASSESSMENT OF THE EFFECTIVENESS OF DIFFERENT HAART COMBINATIONS AT KOMFO ANOKYE TEACHING HOSPITAL
}

\author{
PAA KOFI TAWIAH ADU GYAMFI',2, KWESI BOADU MENSAH ${ }^{*}$, STEPHEN MENSAH ARHIN ${ }^{1}$, ALIU MOOMIN ${ }^{1}$
}

1Department of Pharmacology, Faculty of Pharmacy and Pharmaceutical Science, College of Health Science, Kwame Nkrumah University of Science and Technology, Kumasi, Ghana, ${ }^{2}$ Department of Nursing and Midwifery, Faculty of Health and Allied Sciences, Pentecost

University, Accra, Ghana

Email: kbmensah.pharm@knust.edu.gh

Received: 07 Dec 2019, Revised and Accepted: 30 Jan 2020

\section{ABSTRACT}

Objective: Although all marketed antiretrovirals (ARVs) have proven efficacy, genetic differences can result in varied effectiveness. This study was conducted to determine the effectiveness of different Highly Active Antiretroviral Therapy (HAART) combinations among patients attending HIV clinic at a Major Teaching Hospital in Ghana.

Methods: The study was a retrospective study involving 500 patients at an HIV clinic in the Ashanti Region of Ghana.

Results: Twelve major antiretroviral combinations for HAART were prescribed at the study center. The most prescribed drug combinations were $\mathrm{AZT}+3 \mathrm{TC}+\mathrm{EFV}$ and AZT $+3 \mathrm{TC}+\mathrm{NVP}$. The study identified that HAART, irrespective of the kind of drug combination used, was effective at increasing CD4 count within the first 6 mo of therapy initiation in the study population. However, the magnitude of the increases differed from combination to combination. All HAART combinations with zidovudine as one of the drugs resulted in higher CD4 counts compared with combinations containing stavudine. HAART with nevirapine also resulted in a higher CD4 count than those with efavirenz. However, efavirenz-based combinations appeared to be more effective in critically ill patients and patients with mean CD4+T helper cells count below $100 \mathrm{cell} / \mathrm{mm}^{3}$. More importantly, efavirenz was common among all HAART combinations that resulted in treatment failure.

Conclusion: There was significant variation in response to different HAART combination among Ghanaian HIV patients. However, there was no statistically significant difference in mean CD4 count between the two most predominately used HAART i. e AZT +3TC+EFV and AZT +3TC+NVP.

Keywords: Haart, AIDS, HIV, Antiretrovirals, Effectiveness, Sub-Sahara Africa

(C) 2020 The Authors. Published by Innovare Academic Sciences Pvt Ltd. This is an open-access article under the CC BY license (http://creativecommons.org/licenses/by/4.0/] DOI: http://dx.doi.org/10.22159/ijpps.2020v12i3.36567. Journal homepage: https://innovareacademics.in/journals/index.php/ijpps

\section{INTRODUCTION}

The advent of Highly Active Antiretroviral Therapy has tremendously reduced HIV morbidity and mortality $[1,2]$. Antiretroviral (ARV) are medications for the treatment of infection by retroviruses, primarily HIV. When such medicines, typically three or four, are acquired in combination, the approach is known as Highly Active Antiretroviral Therapy (HAART) [3]. Compared with Antiretroviral (ARV) monotherapy, HAART has significantly reduced treatment failures, suppressed viral transmission and mortality [4]. Although all marketed ARVs have proven efficacy, patient variability as a result of genetic differences in response to drug action can result in varied effectiveness [5]. These variations have been identified as a major problem which can lead to either sub-therapeutic or supratherapeutic treatment outcomes [6]. Pharmacokinetics of a drug can be affected by genetic polymorphism and can therefore lead to reduce plasma and tissue concentration, thereby altering the effectiveness and safety of a prescribed drug [7]. For example, it has been shown that there are variations in pharmacokinetics, efficacy and toxicity of ARVs among people of diverse ethnicity even at standard or recommended doses $[8,9]$.

There has not been any pharmacogenomics studies in Ghanaian population to help tailor antiretroviral drug selection for maximum health benefit of HIV patients. As such, the effectiveness or otherwise of Highly Active Antiretroviral Therapy is yet to be studied comprehensively in Ghana. It is very possible that due to genetic variation as well as some sociocultural practices, Ghanaian HIV patients may not be obtaining the expected benefits associated with HAART. CD4+T helper lymphocyte cell count has been used previously as a predictive measure of development and prognosis of AIDS especially in resource constraint settings where viral load studies may be difficult. We had earlier reported variation in HAART associated adverse effects in Ghanaian population [10]. Therefore, this study was conducted to determine the effectiveness of different HAART combinations among patients attending HIV clinic at Komfo Anokye Teaching Hospital.

\section{MATERIALS AND METHODS}

\section{Study design}

The study was a cross-sectional, descriptive and retrospective in approach. The study was carried out at an HIV clinic at the Komfo Anokye Teaching Hospital in the Ashanti Region of Ghana. Variables were extracted from the patients' folders using a data collection tool and these included age, gender, level of education, occupation, marital status, type of combination therapy, comorbidities, as well as baseline CD4 counts before and 6 mo after initiation of HAART treatment.

\section{Inclusion criteria}

Patients with complete demographic information and documented usage of antiretroviral drugs for at least once at the study site. Patients with CD4 recorded at the start of therapy and at least 6 mo into therapy.

Exclusion criteria

Patients with incomplete medical history and records, and those with incomplete pre-adherence counselling therapy, referred patients, patients with kidney and liver co-morbidities. Patients without information on CD4 cells were also excluded.

\section{Sample size determination}

The minimum sample size was determined based on the formula modified by Naing et al. (2006)

$$
\mathrm{n}=\mathrm{Z} 2 \mathrm{P}(1-\mathrm{P}) / \mathrm{d} 2 .[10] \text {. }
$$


Where $n=$ sample size, $\mathrm{Z}=\mathrm{Z}$ statistic for a level of confidence, $\mathrm{P}=$ expected prevalence (in proportion of one) and $d=$ precision (in proportion of one). Using a $\mathrm{Z}$ statistic for a 95\% confidence level (i.e., $\mathrm{Z}=1.96$ ), precision of $5 \%$ (i.e., $d=0.05$ ) and a prevalence of $3.2 \%$ representing the prevalence of the disease in the Ashanti Region of Ghana [11]

\section{Sampling technique}

Systematic random sampling was used to select patients' folders for the study, covering the periods from 2011 to 2015. A total of one hundred patients were selected from each year of the study, by using a constant number $\left(\mathrm{k}^{\mathrm{th}}\right)$ to select a folder, making a total of 500 folders for the entire five years. The folders were thoroughly examined using the inclusion and exclusion criteria.

\section{Analysis of data}

The data captured was entered into Statistical Package for Social Sciences (SPSS) Version 20.0, examined, cleaned and analyzed. Mean and standard deviation was used to describe continuous variables with normal distribution whiles median and interquartile rage were used to describe continuous variables with skewed distribution. Categorical variables were analyzed using chi-square test. A p-value less than 0.05 was used to assess the level of significance.

\section{Ethical consideration}

Ethical clearance (CHRPE/AP/156/16) was obtained from the Committee on Human Research, Publication and Ethics of the Kwame Nkrumah University of Science and Technology, Kumasi. Permission was obtained from the head of the directorate of Medicine at Komfo Anokye Teaching Hospital before data collection was started. Names and addresses of patients were not recorded to ensure that their identities are not exposed. Case Record Forms were stored in a safe place with restricted access. The computer used for the data entry was password protected and secured and only the principal investigator had access to it. Data obtained was used exclusively for the purposes of this study.

\section{Limitations of the study}

One of the main limitations was the retrospective nature of the study. It was not possible to ensure that all patients complied with their treatment. This could confound the efficacy of the HAART in this study. The use of CD4 as a measure of efficacy instead of viral load was also a major limiting factor due to resource constraints.

\section{RESULTS}

\section{Socio-demographic characteristics}

The mean age of the patients was $39.94 \mathrm{y}$. Most of the respondents were females constituting $70.8 \%(n=354$,$) as against (n=146,29.2$ $\%$ ) of males. Over $70 \%$ of participants had been educated up to the Junior High School or have had no formal education at all. Seventythree percent described themselves as self-employed (see reference 9 for more details).

\section{Drug combination types used by respondents}

Out of the 500 respondents in the study, only 12 of many possible combinations of ARVs were prescribed. Among the 12 combinations, AZT+3TC with either EFV OR NVP accounted for more than 50\%. However, 3TC+TDF+EFV combination was also commonly used a as second line option (see reference 9 for further details).

\section{HAART combinations and CD4}

There was a significant difference between the mean CD4 count before initiation of therapy $(159.7 \pm 118.7)$ and that after 6 mo of initiation of therapy $(344.2 \pm 173.4 \quad(p<0.001))$. This suggested HAART, irrespective of the kind of drug combination used, was able to increase CD4 count within the first 6 mo of therapy initiation within the study population (table 1). However, the magnitude of the increases in CD4 cells differed significantly from combination to combination (table 1).

Table 1: Mean CD4 count compared with the drug combination

\begin{tabular}{ll}
\hline Variable & Mean CD4 count (cells/mm $\mathbf{m}^{3} \pm \mathbf{S D}$ \\
\hline Initial CD4 & $159.7 \pm 118.7$ \\
CD4 after 6 mo & $344.2 \pm 173.4$ \\
ART combinations & \\
AZT,3TC,EFV & $205.39 \pm 125.7$ \\
AZT,3TC,NVP & $220.64 \pm 138.6$ \\
3TC,TDF,EFV & $132.49 \pm 105.2$ \\
D4T,3TC,EFV & $128.68 \pm 129.8$ \\
TDF,EFV,FTC & $141.24 \pm 98.9$ \\
D4T,3TC,NVP & $190.75 \pm 118.1$ \\
3TC,NVP,TDF & $176.00 \pm 156.6$ \\
OTHERS & $112.33 \pm 75.1$ \\
\hline
\end{tabular}

Data expressed as mean \pm SD. N=459. Paired samples T-test was used to compare initial CD4 to CD4 after 6months and One-way ANOVA followed by scheffe's post hoc test was used to compare CD4 amongst the different ARV combination. ${ }^{* * *}$ means $\mathrm{P} \leq 0.0001$
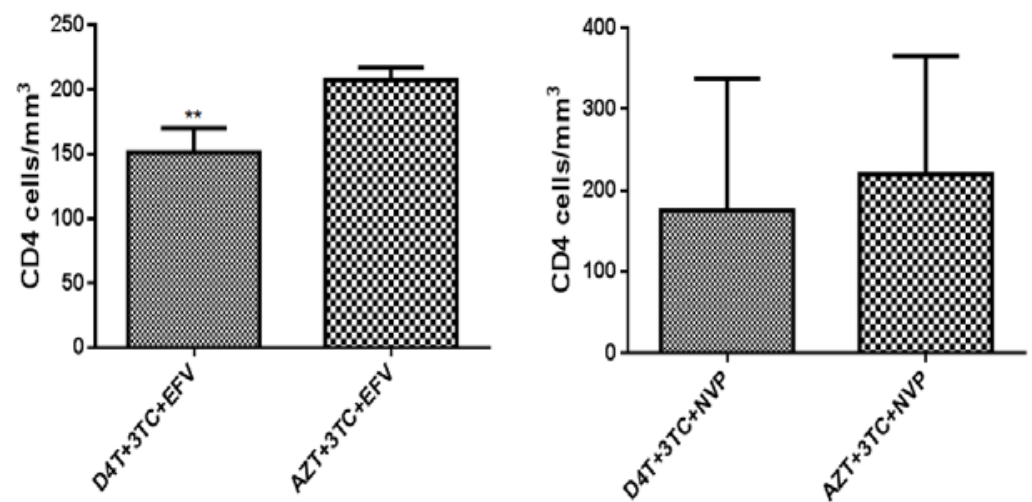

Fig. 1: (a) Comparing AZT+3TC+EFV and D4T+3TC+EFV on CD4 count, **P $\leq 0.001$ using unpaired test, 1(b) Comparing AZT+3TC+NVP and D4T+3TC+NVP on CD4 count 


\section{Comparing mean CD4 of various HAART combinations}

There was no statistically significant difference in CD4 count between the two most predominately used ARV combinations for
HAART i. e AZT, 3TC, EFV and AZT, 3TC, NVP. Interestingly, these two major combinations increased the mean CD4 count significantly when compared to $3 \mathrm{TC} / \mathrm{TDF} / \mathrm{EFV}$ which was also highly preferred by prescribers as a second-line treatment option (table 2).

Table 2: Comparing the mean CD4 of various HAART combination

\begin{tabular}{|c|c|c|}
\hline Combination type & Combination type & Mean difference \\
\hline \multirow[t]{7}{*}{ AZT,3TC,EFV } & AZT,3TC,NVP & -15.25404 \\
\hline & 3TC,TDF,EFV & $72.89443^{*}$ \\
\hline & D4T,3TC,EFV & $76.70674^{*}$ \\
\hline & TDF,EFV,FTC & 64.15145 \\
\hline & D4T,3TC,NVP & 14.63674 \\
\hline & 3TC,NVP,TDF & 29.38674 \\
\hline & OTHERS & 93.05341 \\
\hline \multirow[t]{6}{*}{ AZT,3TC,NVP } & 3TC,TDF,EFV & $88.14847 *$ \\
\hline & D4T,3TC,EFV & $91.96078^{*}$ \\
\hline & TDF,EFV,FTC & 79.40548 \\
\hline & D4T,3TC,NVP & 29.89078 \\
\hline & 3TC,NVP,TDF & 44.64078 \\
\hline & OTHERS & 108.30744 \\
\hline \multirow[t]{5}{*}{ 3TC,TDF,EFV } & D4T,3TC,EFV & 3.81231 \\
\hline & TDF,EFV,FTC & -8.74299 \\
\hline & D4T,3TC,NVP & -58.25769 \\
\hline & 3TC,NVP,TDF & -43.50769 \\
\hline & OTHERS & 20.15897 \\
\hline \multirow[t]{4}{*}{ D4T,3TC,EFV } & TDF,EFV,FTC & -12.55529 \\
\hline & D4T,3TC,NVP & -62.07000 \\
\hline & 3TC,NVP,TDF & -47.32000 \\
\hline & OTHERS & 16.34667 \\
\hline
\end{tabular}

All data are expressed as mean \pm SD $(n=459),{ }^{*} p<0.05$, using (one-way ANOVA followed by Scheffe’s post hoc test).

Comparing zidovudine (AZT) based combinations to stavudine (D4T) base combination

For all HAART combinations which differed only in either AZT or D4T; AZT combinations consistently resulted in higher mean CD4 counts compared with D4T combinations (fig. 1a and b). This observation was statistically significant when AZT+3TC+EFV was compared to D4T+3TC+EFV but not when nevirapine (NVP) replaced efavirenz (EFV). It appears that AZT synergizes with EFV or a combination of 3TC+EFV in Ghanaian HIV patients.
Comparing Efevirenz (EFV) based combinations to nevirapine (NVP) combinations

Generally, the study showed that NVP improved patient baseline CD4 count across all combinations better than EFV although not statistically significant (fig. 2a), however in patients with CD4 count lower than $100 \mathrm{cell} / \mathrm{mm}^{3}$, it was evident that EFV was more effective in raising CD4 count better than NVP (fig. 2b). EFV was found in all HAART regimen associated with treatment failure.
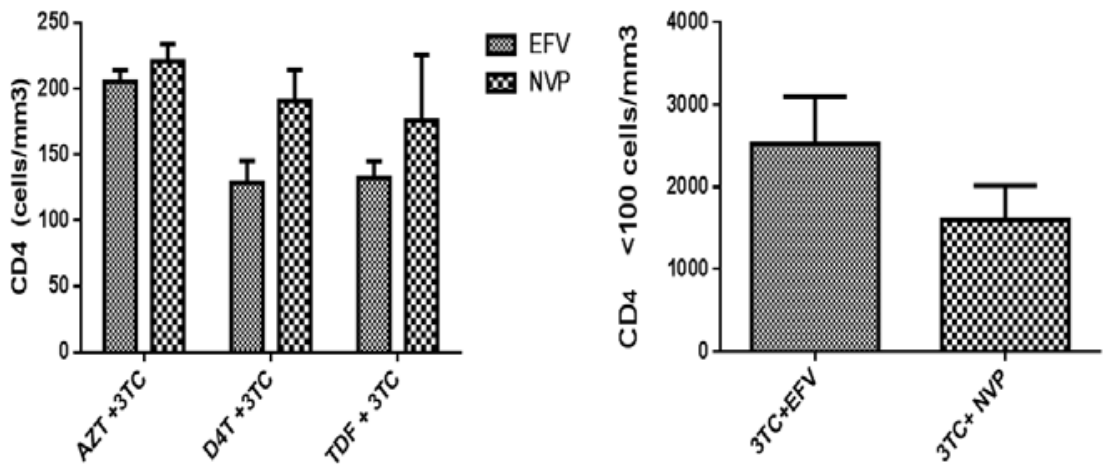

Fig. 2: a. Comparing HAART regimen that differed only in either efevirenz or Nevirapine for 6 mo on CD4 cell count, b. Comparing CD4 cell change of patients with low $\mathrm{CD} 4$ count $\left(100 \mathrm{cell} / \mathrm{mm}^{3}\right)$ treated with either efevirenz or Nevirapine combinations for 6 consecutive months

\section{DISCUSSION}

This study highlighted the major HAART combinations and their effectiveness in Ghanaian population. The most prescribed combinations were $\mathrm{AZT}+3 \mathrm{TC}+\mathrm{EFV}$ and $\mathrm{AZT}+3 \mathrm{TC}+\mathrm{NVP}$. This combination is similar to what has been reported elsewhere $[12,13]$. A study conducted in KATH also confirmed AZT+3TC+EFV was the most commonly prescribed HAART followed by AZT+3TC+NVP [13] These combinations were in line with the recommendations of the
National guidelines for the use of ARV in Ghana as well as WHO guidelines [14].

There was a significant rise in mean CD4 count after 6 mo of initiation of therapy. $\mathrm{Kr}$ et al., (2014) also reported improvement in CD4 cell count within 6 mo of therapy in HIV patients as well as in those with HIV comorbidity [15]. It is prudent for HIV/AIDS patients to receive HAART regardless of adverse effects. It has been documented that the degree of immune restoration is dependent on 
the degree of immunodeficiency at the initiation of HAART treatment [16]. This may affect both morbidity and mortality rates in HIV patients.

The mean CD4 count before initiation of therapy in this study was 159.7 cells $/ \mathrm{mm}^{3}$. This shows that most of the diagnoses occurred after patients reported clinical symptoms. Several studies have reported that initiating HAART therapy early when CD4 count is high results in higher mean CD4 levels and better treatment outcomes $[17,18]$. The most essential prognosis indicator of clinical advancement and survival after HAART initiation is based on CD4 count and medication adherence [19]. Patients with lower baseline CD4 count remain at risk for opportunistic infections for a substantially longer period than patients starting HAART at higher CD4 counts hence affecting risk for serious morbidity and death [20-23].

A study conducted in high-income countries suggests that EFV is superior to NVP in achieving an undetectable viral load [24]. In this study, however, NVP proved to be more effective in improving CD4 counts than EFV although not statistically significant. Again, in all combinations that differed only in AZT and D4T, AZT patients experienced significant improvement in CD4 and less adverse effects. Subsequently, there were low CD4 count increase and high adverse effects with D4T and NVP combinations. Interestingly, when CD4 was very low and in critically ill patients, EFV proved better than NVP at increasing CD4 counts. However, EFV HAART combination was associated with treatment failures or immunologic failure during HIV management. This result conflicts with earlier reports by Van Oosterhout et al. [25].

AZT efficacy was remarkably consistent in all the HAART combination. It appeared that AZT synergies with EFV but its use was always associated with severe anemia, which led to a change in the drug combination in certain instances. A prospective cohort study conducted by Shet et al., 2014, indicated that patients on zidovudine had 22 times higher risk of developing anemia compared to those on other regimens [26]. However, adverse drug reactions (ADRs) associated with the use of zidovudine is expected to increase because of its proven efficacy in Ghanaian HIV patients and its use in mother to child transmission [27].

Contrary to the perception of drug stock out as the more important reason for drug change, this study found that drug toxicity was rather the most important reason for medication change. This is in line with other reports $[26,28]$. It emerged from the study that TDF+3TC+EFV was becoming a popular treatment option and second line drug when drug toxicity was of major concern. However a careful analysis showed that it was less efficacious at improving CD4 when compared to traditional options of AZT+3TC with either EFV or NVP within the first 6 mo of initiation of therapy.

\section{CONCLUSION}

There were 12 major antiretroviral combinations that were employed in HAART for the treatment of HIV patients in the study. All HAART therapy resulted in statistically significant increases in mean CD4 counts within 6 mo of initiation of therapy. However, the magnitude of the increases differed from combination to combination.

\section{ABBREVIATION}

Antiretrovirals (ARVs), Human Immunodeficiency Virus (HIV), CD4+T helper cells (CD4 cells), Stavudine (D4T); Lamivudine (3TC); zidovudine (AZT); Efavirenz (EFV); Tenofovir (TDF); Lopinavir/ritonavir (LPV/r); Abacavir (ABC); Emtricitabine (FTC), Zidovudine (AZT).

\section{ACKNOWLEDGEMENT}

Authors are grateful to all staff of Komfo Anokye Teaching hospital HIV clinic for their assistance during the study.

\section{FUNDING}

Nil

\section{AUTHOR CONTRIBUTIONS}

PKT and KBM developed concept. PKT and MA did data collection and analysis. SMA wrote the first manuscript draft.

\section{CONFLICT OF INTERESTS}

Authors have no competing interests to declare.

\section{REFERENCES}

1. Zhang F, Dou Z, Yu L, Xu J, Jiao JH, Wang N, et al. The effect of highly active antiretroviral therapy on mortality among HIVinfected former plasma donors in China. Clin Infectious Diseases 2008;47:825-33.

2. Nsagha DS, Weledji EP, Assob NJ, Njunda LA, Tanue EA, Ayima CW, et al. Highly active antiretroviral therapy and dyslipidemia in people living with HIV/AIDS in Fako Division, South West Region of Cameroon. BMC Cardiovascular Disorders 2015;15:95.

3. Bennett DE, Bertagnolio S, Sutherland D, Gilks CF. The world health organization's global strategy for the prevention and assessment of HIV drug resistance. Antiviral Therapy 2008; Suppl 2:1-13.

4. Braitstein P, Brinkhof MW, Dabis F, Schechter M, Boulle A, Miotti $\mathrm{P}$, et al. Mortality of HIV-1-infected patients in the first year of antiretroviral therapy: comparison between lowincome and high-income countries. Lancet 2006;367:817-24.

5. Ma Q, Lu AY. Pharmacogenetics, pharmacogenomics, and individualized medicine. Pharmacol Rev 2011;63:437-59.

6. Tozzi V. Pharmacogenetics of antiretrovirals. Antiviral Res 2010;85:190-200.

7. Ingelman Sundberg M, Sim SC, Gomez A, Rodriguez Antona C. Influence of cytochrome P450 polymorphisms on drug therapies: pharmacogenetic, pharmacoepigenetic and clinical aspects. Pharmacol Ther 2007;116:496-526.

8. Pavlos R, Phillips EJ. Individualization of antiretroviral therapy. Pharmacogenomics Pers Med 2012;5:1.

9. Alfonso V, Bermbach N, Geller J, Montaner JS. Individual variability in barriers affecting people's decision to take HAART: a qualitative study identifying barriers to being on HAART. AIDS Patient Care STDs 2006;20:848-57.

10. Mensah KB, Adu Gyamfi PK, Boakye Gyasi E. HAART therapy in ghana: assessment of adverse drug reaction reports of patients at an HIV clinic and a teaching hospital. J Basic Clin Pharm 2017;8:1.

11. Naing L, Winn T, Rusli BN. Practical issues in calculating the sample size for prevalence studies. Arch Orofacial Sci 2006;1:9-14

12. Ghana Statistical Service. 2010 population and housing census report. Ghana Statistical Service; 2014.

13. Sandeep B, Chavan VR, Raghunandan M, Arshad M, Sayana SB. Factors influencing the substitution of antiretroviral therapy in human immunodeficiency virus/acquired immunodeficiency syndrome patients on first-line highly active antiretroviral therapy. Asian J Pharm Clin Res 2014;7:117-20.

14. Obeng RK. Prevalence and causes of antiretroviral treatment failure among adults receiving therapy at Komfo Anokye teaching hospital (Doctoral dissertation); 2010.

15. World Health Organization. Antiretroviral therapy of HIV infection in infants and children: towards universal access: recommendations for a public health approach-2010 revision. World Health Organization; 2010.

16. Kr D, Sanji N, Aggarwal V, Sr R, Hs S. Changes in the CD4 counts, hemoglobin and weight in patients with HIV alone and HIV-TB co-infection. Asian J Pharm Clin Res 2014;7:35-8.

17. Mayer $\mathrm{KH}$, Cooney EL. Clinical indicators of immune restoration following highly active antiretroviral therapy. Clin Infectious Diseases 2002;34:224-33.

18. Insight Start Study Group. Initiation of antiretroviral therapy in early asymptomatic HIV infection. New England J Med 2015;373:795-807.

19. Violari A, Cotton MF, Gibb DM, Babiker AG, Steyn J, Madhi SA, et al. Early antiretroviral therapy and mortality among HIVinfected infants. New England J Med 2008;359:2233-44.

20. Barasa SS. True story about HIV: theory of viral sequestration and reserve infection. HIV/AIDS (Auckland, NZ) 2011;3:125.

21. Ferradini L, Jeannin A, Pinoges L, Izopet J, Odhiambo D, Mankhambo L, et al. Scaling up of highly active antiretroviral therapy in a rural district of Malawi: an effectiveness assessment. Lancet 2006;367:1335-42. 
22. Lawn SD, Myer L, Orrell C, Bekker LG, Wood R. Early mortality among adults accessing a community-based antiretroviral service in South Africa: implications for programme design. AIDS 2005;19:2141-8

23. Bonnet F, Thiebaut R, Chene G, Neau D, Pellegrin JL, Mercie P, et $a l$. Determinants of clinical progression in antiretroviral-naïve HIV-infected patients starting highly active antiretroviral therapy. Aquitaine Cohort France 1996-2002. HIV Med 2005;6:198-205.

24. Egger M, May M, Chene G, Phillips AN, Ledergerber B, Dabis F, et al. Prognosis of HIV-1-infected patients starting highly active antiretroviral therapy: a collaborative analysis of prospective studies. Lancet 2002;360:119-29.

25. Pillay P, Ford N, Shubber Z, Ferrand RA. Outcomes for efavirenz versus nevirapine-containing regimens for treatment of HIV-1 infection: a systematic review and meta-analysis. PloS One 2013;8:e68995.
26. Van Oosterhout JJ, Bodasing N, Kumwenda JJ, Nyirenda C, Mallewa J, Cleary PR, et al. Evaluation of antiretroviral therapy results in a resource-poor setting in Blantyre, Malawi. Trop Med Int Health 2005;10:464-70.

27. Shet A, Antony J, Arumugam K, Dodderi SK, Rodrigues R, DeCosta A. Influence of adverse drug reactions on treatment success: a prospective cohort analysis of HIV-infected individuals initiating first-line antiretroviral therapy in India. PLOS ONE 2014;9:e91028.

28. Murphy EL, Collier AC, Kalish LA, Assmann SF, Para MF, Flanigan TP, et al. Highly active antiretroviral therapy decreases mortality and morbidity in patients with advanced HIV disease. Annals Internal Med 2001;135:17-26.

29. Hart E, Curtis H, Wilkins E, Johnson M. National review of first treatment change after starting highly active antiretroviral therapy in antiretroviral-naïve patients. HIV Med 2007;8:186-91. 